\title{
Genetic variants related to nicotine dependence
}

\author{
B. Corradini ${ }^{\text {a }}$, P. Sánchez-Diz ${ }^{\text {b,c }}$, M. Alù ${ }^{\text {a }}$, A. Estany-Gestal ${ }^{\text {d,e }}$, A. Carracedo ${ }^{\text {b,c }}$, G. Ferri ${ }^{\text {a,* }}$ \\ ${ }^{a}$ Department of Diagnostic and Laboratory Service and Legal Medicine, Section of Legal Medicine, University of Modena and Reggio Emilia, Italy \\ ${ }^{\mathrm{b}}$ Institute of Forensic Sciences Luis Concheiro, Genomics Medicine Group, University of Santiago de Compostela, Spain \\ ${ }^{\mathrm{c}}$ Centro de Investigación Biomédica en Enfermedades Raras (CIBERER), Spain \\ ${ }^{\mathrm{d}}$ Department of Preventive Medicine and Public Health, University of Santiago de Compostela, Santiago de Compostela, Spain \\ ${ }^{\mathrm{e}}$ Foundation for Research, Development and Innovation of the University Hospital of Santiago (IDICHUS), Santiago de Compostela, Spain
}

\section{A R T I C L E I N F O}

Article history:

Received 19 September 2011

Accepted 30 September 2011

\section{Keywords:}

Smoking

Addiction

Genetic association

Nicotine dependence

SNP

\begin{abstract}
A B S T R A C T
Large-scale population studies have proved that genetic factors contribute to individual differences in smoking behavior. Genes responsible for nicotine's pharmacokinetics and pharmacodynamics seem mainly involved, although a significant fraction of variance remains unexplained. In this study we examined 10 SNPs from 8 candidate genes with positive previous reports of association with smoking. A total of 454 Italian unrelated subjects were genotyped by a multiplex minisequencing assay through the SNaPShot kit. Cases were chosen as current and former nicotine dependent (FTND $\geq 4$ and SQ $\geq 15$ ), while controls were smoking-exposed but non-dependent and never smoker individuals (FTND $=0$ and $\mathrm{SQ} \leq 10$ and $\mathrm{FTND}=0$ and $\mathrm{SQ}=0$, respectively). Preliminary results shows that the SNPs CHRNA5rs16969968 and CHRNA3-rs1051730 could be associated with risk of developing nicotine dependence. Factors as age, sex, and exposition to smoke were also found as possible factors of risk of nicotine addiction. The identification of susceptibility loci for individual response to substance abuse is particularly motivating for medicine for the global epidemic dimension of addictions and the urgent need of effective preventive and therapeutic strategies.
\end{abstract}

() 2011 Elsevier Ireland Ltd. All rights reserved.

\section{Introduction}

Substance abuse disorders represent a significant burden on worldwide health care system and not least permeating each branch of forensic medicine. Among these, cigarette smoking has a deleterious impact on society being one of the largest cause of morbidity and premature deaths in western countries. Despite this awareness, about $22 \%$ of the world population currently consumes tobacco [1]. Smoking behavior is a complex trait which involves key behavioral steps from the initial use to a chronic dependence condition, each of which is influenced by the interplay between environmental factors and a documented genetic background. Recent advances in the field through association studies and GWAS approaches attributed the strongest genetic influence to the nicotinic acetylcholine receptors and metabolizing genes, making promising candidates as predictive markers [2]. Even with considerable progress, a significant fraction of the estimated heritability (40-60\%) as well as the biological mechanism underlying the correlation with the disease remain unexplained

\footnotetext{
* Corresponding author.

E-mail address: gianmarco.ferri@unimore.it (G. Ferri).
}

with a plausible involvement of several chromosome regions throughout the genome [2].

\section{Materials and methods}

The study population consisted of 454 unrelated volunteers of Italian origin who have been measured for the degree of nicotine dependence (ND) with the Fagerström Test for Nicotine Dependence (FTND) (scores 0-10) and for the Smoking Quantity (SQ) per day. We considered as cases those subjects who show nicotine dependence (ND) according to FTND and SQ values (FTND $\geq 4$ and $\mathrm{SQ} \geq 15$ cigarettes/day), which were classified as current (exposed) and former (non-exposed) smokers. Controls were those individuals displaying not nicotine dependence, sorted as: exposed to tobacco (FTND $=0$ and $\mathrm{SQ} \leq 10$ cigarettes/day) and not exposed (FTND $=0$ and $\mathrm{SQ}=0$ ). Genomic DNA was extracted from buccal swabs using standard Chelex method. A set of 10 autosomal SNPs (rs2273504, rs806368, rs2023239, rs806380, rs324420, rs2184026, rs16969968, rs1051730, rs4950, rs686) linked to different brain systems (CHRN4, CNR1, FAAH, GABAB2, CHRNA5, CHRNA3, CHRNB3, DRD1) was chosen based on positive previous reports of association with smoking [3] or other drugs types [4]. SNPs were included in a multiplex PCR using Qiagen Multiplex kit. Single base extension (SBE) reaction was accomplished by 
SNaPshot approach and products were separated by capillary electrophoresis on ABI PRISM3130 HID Genetic Analyzer. Fragments were analyzed with GeneMapper ID v 3.2 software. The descriptive analysis, genotype frequencies, the assessing of deviations from Hardy-Weinberg equilibrium and the linkage disequilibrium test were performed with both $\mathrm{R}$ project (v2.12.2) and SNPassoc package, which is at the same time implemented in R. To identify the potential confounding variables, a bivariate analysis was carried out. Those variables with a $p$-value $\leq 0.2$ were considered in the multivariate analysis. The independent variables with the highest level of statistical significance were successively eliminated from the original model, following the AIC criterion and using the glm $\mathrm{R}$ package.

\section{Results}

Main results of the descriptive analysis showed that the mean ages were $37.5( \pm 10.5)$ years in cases and $30.9( \pm 8.6)$ in controls. Likewise, $60.3 \%$ of the cases were men, while men in controls were 40.5\%. Deviations from HW expectations were found in three SNPs, DRD1 in cases, CNR1-rs806380 in controls and CNR1-rs2023239 in both groups ( $p$-values $<0.005$ ); however, further analysis are needed to make reliable conclusions about the observed deviations. Moreover, $p$-values from LD test were significant for the majority of the SNPs pairs ( $p$-value $<0.0001$ ), being GABAB2-CNR1 the only pair with a not significant $p$-value $(>0.165)$. Anyway, as it was mentioned above, conclusions must be made after additional analysis will be performed. Preliminary results from the multivariate model reveals that the SNPs rs2023239, rs16969968 and rs1051730, located in the genes CNR1, CHRNA5 and CHRNA3, respectively, could be associated with risk of developing nicotine dependence. Factors as age, sex, and exposition to smoke were also found as possible risk factors for developing nicotine addiction. However, CNR1-rs2023239 show significant deviations from HWE expectations both in the cases sample and also in controls, therefore this latter result was deemed unusable for now.

\section{Discussion}

Psychopharmacological effects of nicotine are mediated primarily by nicotinic cholinergic receptors (nAChRs). Genes encoding subunits have been proposed as plausible candidates for ND and several have been investigated [2].

Large scale GWAS meta-analyses established association with CHRNA5-CHRNA3-CHRNB4 gene cluster (chromosome 15q2425.1) with smoking severity, dependence and lung cancer.

Specifically, the non-synonymous SNP CHRNA5-rs16969968 and SNP CHRNA3-rs1051730 are the most appealing candidates.
Furthermore, rs1051730 is already part of the so-called "personal genomics" through a direct-to-consumer testing powered by an Icelandic company to learn your own risk for developing ND [5], even if the predictive ability is still limited. In line with the literature, in our population the group of cases (FTND $\geq 4$ ) was more likely to hold the rs16969968-A allele and rs1051730-T allele comparing with the control group without dependence $(\mathrm{FTND}=0$ ).

For both markers the risk alleles were the same as previously reported [5]. The other analyzed variants did not show significant differences in frequency distributions. This could also indicate insufficient statistical power to detect small effects of single polymorphisms in our sample.

The knowledge of molecular components of complex traits such as response to drugs, addictive disorders, personality and phenotypic traits are also entering into the sensitive forensic field [6]. Current expectations concerning rapid advances in the neurobiological basis of relevant behaviors are accompanied by a heated discussion on implication for social institutions and the justice system $[7,8]$. Identification of genetic factors affecting substance dependencies is a major interest of medicine today in assessing individual vulnerability and in developing more effective interventions, though multifactorial etiology of addictions needs to invoke the use of great caution within risk inference.

\section{Conflict of interest statement}

None.

\section{References}

[1] D.K. Hatsukami, L.F. Stead, P.C. Gupta, Tobacco addiction, Lancet 371 (2008) 20272038.

[2] L.J. Bierut, Genetic vulnerability and susceptibility to substance dependence, Neuron 69 (2011) 618-627.

[3] N. Saccone, S.F. Saccone, A.L. Hinrichs, J.A. Stitzel, et al., Multiple distinct risk loci for nicotine dependence identified by dense coverage of the complete family of nicotinic receptor subunit (CHRN) genes, Am. J. Med. Genet. B Neuropsychiatr. Genet. 150 (2009) 453-466.

[4] A. Agrawal, L. Wetherill, D.M. Dickk, et al., Evidence for association between polymorphisms in the cannabinoid receptor 1 (CNR1) gene and cannabis dependence, Am. J. Med. Genet. B Neuropsychiatr. Genet. 150 (2009) 736-740.

[5] T.E. Thorgeirsson, F. Geller, P. Sulem, T. Rafnar, A. Wiste, et al., A variant associated with nicotine dependence, lung cancer and peripheral arterial disease, Nature 452 (2008) 638-642.

[6] M. Kayser, P.M. Schneider, DNA-based prediction of human externally visible characteristics in forensics: motivations, scientific challenges, and ethical considerations, Forensic Sci. Int. Genet. 3 (2009) 154-161.

[7] M.T. Treadway, J.W. Buckholtz, On the use and misuse of genomic and neuroimaging science in forensic psychiatry: current roles and future directions, Child Adolesc. Psychiatr. Clin. N. Am. 20 (2011) 533-546.

[8] F. Forzano, P. Borry, A. Cambon-Thomsen, S.V. Hodgson, A. Tibben, P. deVries, C. van El, C. Cornel, Italian appeal court: a genetic predisposition to commit murder, Eur. J. Hum. Genet. 18 (2010) 519-521. 